\title{
An IBIS-based model to support group discussions
}

\author{
G. Bellassai ${ }^{1}$ \\ Universidad Católica de Asunción, Paraguay \\ M. Borges ${ }^{2}$ \\ Santa Clara University, USA* \\ D. A. Fuller ${ }^{3}$ \\ Pontificia Universidad Católica de Chile, Chile

\section{J. A. Pino 4} \\ Universidad de Chile, Chile
}
A. C. Salgado5
Universidade Federal de Pernambuco, Brazil

\begin{abstract}
In this paper, an extension of the IBIS argumentation model is presented to support pre-meeting activities. The data model of a system called SISCO is introduced, and a case example is presented. This system allows remote and asynchronous collaboration to do the work of meeting preparation.
\end{abstract}

CSCW, IBIS, pre-meetings.

\section{Keywords}

\footnotetext{
${ }^{1}$ Laboratório de Electrónica Digital, Asunción, Paraguay, gbellas@ledip.py

2 Object Technology Laboratory, School of Engineering, Santa Clara, CA 95053, USA, fax: + 1408 554-5474, Phone: +1 408 554-2139, mborges@otl.scu.edu

* On leave from:Universidade Federal do Rio de Janeiro, Brazil., mborges@nce.ufrj.br

${ }^{3}$ Depto. de Ciencia de la Computación, Casilla 306, Santiago 22, Chile, dfuller@ing.puc.cl

${ }^{4}$ Depto. de Ciencias de la Computación, Casilla 2777, Santiago, Chile, jpino@dcc.uchile.cl

5 Departamento de Informática, Recife, Brazil, acs@di.ufpe.br
} 


\section{INTRODUCTION}

The use of computer systems to intermediate and facilitate human interaction has been expanding very fast. CSCW (Computer Supported Cooperative Work) is an emergent area that examines how computers can assist several people working together (Khoshafian and Buckiewicz, 1995). In its various proposals, people can be at the same or different places. It also matters when the human interactions are synchronous (same time) or asynchronous (different time). Among the main applications developed so far we can mention meetings support, workflow, and cooperative editors.

The support of meetings is very appealing for business in general. Systems that support meetings aim to increase efficiency by speeding the decision process and by generating automatic documentation. Besides, if the meeting is geographically distributed there is also a reduction of traveling costs and time.

Several systems have been designed to support meetings on their various forms (Nunamaker et al., 1991). An alternative idea is to provide a tool for the preparation of a pre-meeting (Bellassai, Borges, Fuller, Pino and Salgado, 1995).

In SISCO we provide a discussion environment where no decisions are made. Many times people are not prepared to discuss or, in some cases do not know about, the agenda items of the meeting they are invited to. This fact can produce very long and inefficient meetings. The purpose is to improve meetings productivity and to well inform meetings participants before the face-to-face meeting takes place. In SISCO we want to make meetings more efficient but we do not attempt to eliminate them because we believe that face-to-face meetings are essential for decision making.

To support group interaction such as in SISCO it is necessary to consider the varied aspects of collaboration. They include information sharing, communication among members of a group, and coordination of their cooperative activities.

In any group interaction there is a need for a repository of data. If all members are present, such as in a face-to-face meeting, the repository works more as a group memory, to where one can recall whenever it is needed to review topics discussed in the meeting. In this case however, it is assumed that a member is aware of the main elements of the discussion. On the other hand, if the interaction is asynchronous, the data repository plays a more active and comprehensive role. Besides playing the role of a group memory, the repository also serves as the mechanism for interaction and awareness to where group members recall when they want to update their knowledge of the meeting status.

The objective of this paper is to present a data model to support group discussions in a premeeting context assuming an asynchronous and geographically distributed interaction. The proposed data model is an extension of the basic IBIS argumentation model (Kunz and Rittel, 1970). The extension includes several elements to facilitate interaction and understanding of the discussion.

The paper is organized as follows. Section 2 presents some requirements for group discussion. The IBIS data model, with its characteristics and deficiencies, is discussed in Section 3. In Section 4 we present our proposed data model. An example illustrates our proposal in Section 5 and the conclusions are presented in Section 6.

\section{GROUP DISCUSSIONS AND PRE-MEETINGS}

Meetings are certainly an important activity people do within organizations (Jay, 1976), (Hackman and Kaplan, 1974) and a recent survey confirms that white-collar workers spend an average of 50\% of their time in these meetings. Jay (Jay, 1976:43) adequately states that "a meeting still performs 
functions that will never be taken over by telephones, teleprinters, Xerox copiers, tape recorders, television monitors, or any other technological instruments of the information revolution".

This is not equivalent to saying that computers are useless for supporting meetings within organizations. Research has been done, for instance, in having face-to-face meetings with interconnected computers (Wagner, Wynne and Mennecke, 1993) and meetings done remotely with the help of multimedia systems (Streitz, Geibler, Haake and Hol, 1994) (Bergmann and Mudge, 1994).

Why meetings are so important? Jay (Jay, 1976) has identified six functions meetings perform, including group membership delimitation (who belongs to the group?), common knowledge, understanding of the collective aim of the group, and be a status arena. These functions are not directly tied to the subject of the meeting but are of a socio-psychological flavor.

Our approach recognizes the role of face-to-face meetings, especially for decision-making. We emphasize the preparation of the meetings with information technology tools as a way to make them effective and efficient.

The preparation of the meeting is crucial in various respects. First of all, it may help to make all members of the group gather valuable information. As Jay puts it: "a solid basis of agreed-on facts is the best foundation to build any decision on" (Jay, 1976:43).

Secondly, and even more significantly, a clarifying discussion held before the meeting may help to reduce both the equivocality and uncertainty of the meeting (Daft and Lengel, 1986). Equivocality in this case refers to the ambiguity and confusion concerning the agenda items: many times meeting members do not know or agree on answers to very basic questions, such as, why are we concerned with this matter?, what are the implications of this matter?, is this the right time to decide on this issue?, what is this matter, anyway?

Uncertainty refers to the lack of answers to questions and issues that need further elucidation, although there is already an understanding about their importance. Of course, meeting members do not identify the nature of their concern. In normal meetings, they just manifest them. But progress in the meeting will probably be reduced, reflecting the deep weakness in the common understanding, especially if most doubts are of the equivocality type.

A discussion held before a meeting then opens interesting opportunities for people to have a sound agreement on many aspects of the meeting. Our goal is to materialize such opportunities by facilitating the means of achieving the interchange of information, opinions, goals, etc. Is a face-toface encounter needed for such interchange? It may be, but it can be complemented with work from everyone's office if a structured, simple-to-use mechanism is available. A distributed software system presents the benefits of keeping the familiar furniture plus easy access to physical and computerized files, and not requiring same-time availability (Ellis, Gibbs and Rein, 1991). Furthermore, if the discussion is done with computer help, it may be stored for later reference.

The social environment of the meeting is very important. If people do not want to collaborate with each other, there is no computer-based system which compulsively will make them effectively work together. We do not address the noncollaboration issue in this paper. Our assumption is that the group members will not try to hide information and have an open, collaborative approach to the group work. This environment is called "Le Canton style of cooperation" and is further discussed in a previous paper (Bellassai, Borges, Fuller, Pino and Salgado, 1995).

Will there be incentives to spend time with the computer simply preparing a meeting? In reality, users interact with the computer but also with the other users in our approach (Bellassai, Borges, Fuller, Pino and Salgado, 1995), and that could make a difference to some users. Also, we expect that the mere need to keep updated will motivate people to connect to the system to see what is new. 
Finally, the system includes features to allow users to stimulate participation in the discussions through virtual rewards.

\section{THE IBIS MODEL OF ARGUMENTATION}

A constructive discussion consists of presenting ideas in an organized way with some supporting arguments that can be understood and revised by other members. In face-to-face meetings people use natural language to express their positions. Although rich by nature, a text or a speech are too ambiguous when not properly organized.

The organization of speech or text will depend very much on the capacity of the individual to put his ideas in simple, concise and objective ways. Even when this goal is attained, due to the various possibilities of speech organization, it may be difficult for another individual to separate the various elements of the speech in order to better understand it. If the speech is meant to be stored by a system, automatic interpretation and classification is even harder.

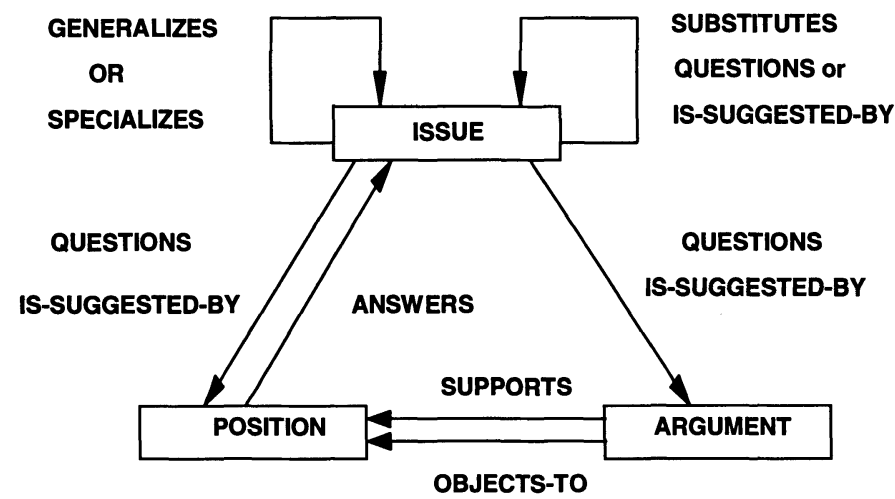

Figure 1 The IBIS Model (reproduced from Kunz, W. and Rittel, H. (1970)).

For example, a speech can mix several elements in different places. Sometimes it is difficult to recognize even more basic issues, like for example, if he is in favor or against a proposal.

In order to help organizing the discussion memory, authors suggest some classification on the statement, by separating it into different categories. In this way it is also possible to save time to introduce the subject. It is always possible that a participant may wrongly classify his statement or mix several categories under the same, like in a free speech. With the model however, one can always check and if it is the case, divide the statement in several parts to better represent the idea.

In 1970, Kunz and Rittel proposed a model called IBIS (Issue based information system) composed of three elements and nine relationships (Kunz and Rittel,. 1970). The model aimed to represent the main elements of a discussion allowing people to understand and easing additional contributions. The conceptual IBIS schema proposed by Kunz and Rittel is depicted in Figure 1. The IBIS model has been successfully applied in discussions (Conklin and Begeman, 1988). The main advantage of the model is its simplicity and intuitiveness. 
In this way, an individual trying to put an argument to a position can write directly the argument text and link to the appropriate position with one of the possible relationships, that is, in-favor or against. The member is restricted to these three types of statements and the mutually exclusive nine relationships. Only a new issue can be inserted without any relationship. All others must take part in one relationship.

For the purpose of our system, we decided to extend the IBIS model to accommodate new types of statements. Each of these statements is explained below. On the other hand, we simplified the relationships by removing some of them.

\section{- Participant}

We defined the concept of a discussion participant in order to identify the origin of the statements. However, we also provide anonymity as the relationships between participant and statements are optional. We have also defined the specialization of a participant related to the role of coordinator;

\section{- Pre-Decision}

Although we decided not to make any decision during the pre-meeting, we thought it would be important to store decisions of previous meetings or assertions to a discussion in the form of pre-decisions. Pre-decisions can in some cases take the form of constraints.

- Proposal

The proposal is a special kind of issue that is suggesting an action, such as the performing of a task, or a re-definition to the issue.

- Task

As a result of a discussion we sometimes need to gather additional information to endorse or to help an argument. A task is the object that represents this job.

\section{- Remark}

We felt that in a discussion there is a need for statements that cannot be classified by any of the IBIS elements. Therefore, in order to avoid distorting the basic elements we create the remark element that can accommodate any statement that does not fit in the original IBIS classification.

\section{OUR PROPOSAL - THE DATA MODEL}

In our approach, the central problem is to manage the discussion among participants in the preparation for a meeting, that means a pre-meeting. A common organized memory - called the discussion database - is provided to assure all participants can have access at any time to discussion elements.

Our database model is an extension of the basic IBIS argumentation model. It has the same basic elements: issue, position and argument; but it is enlarged with remarks and tasks. The data model also includes information about all participants, pointing out the coordinator of the pre-meeting, the agenda items with its objectives and some pre-defined constraints. It is further assumed that one can consult information outside the discussion process in an external repository. 


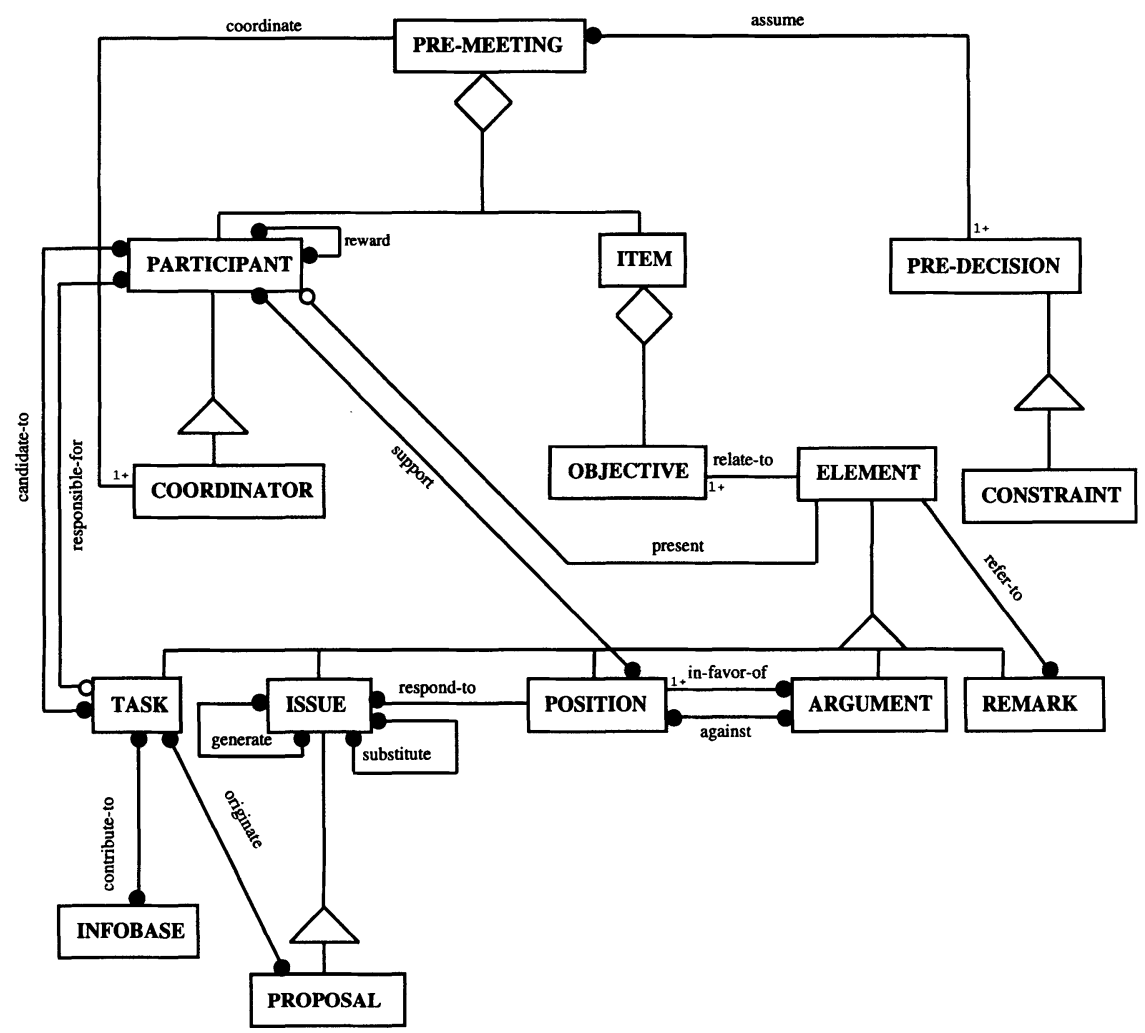

Figure 2 The SISCO data model.

Some basic assumptions are necessary to best define the data model. They are:

- A pre-meeting is a preparation for a meeting, where no decisions, concerning agenda items, are taken.

- At a creation of a pre-meeting, a coordinator is assigned. The coordinator specifies the list of participants, initial pre-decisions, constraints, agenda items and objectives.

- The pre-meeting process takes place with participants asynchronously interacting through the presentation of assertions (issues, positions, arguments and remarks).

- Some issues are classified as proposals and these may suggest a task to be performed.

- Participants may candidate themselves to carry out a task. One of these candidates is chosen by the coordinator to be responsible for the task.

- Tasks have deadlines that can only be changed by the coordinator.

The data model was completely specified using the Object Modeling Technique (OMT) (Rumbaugh et al., 1991). The relationship among the object classes defined are illustrated in Figure 2. In this methodology, aggregations are drawn like associations with a small diamond indicating the assembly end of the relationship and the notation for generalization is a triangle connecting a superclass to its 
subclasses. In what follows, the data model is described including the object classes, the relationships among them, some constraints, and the operations concerned.

\subsection{Description of object classes}

The basic class of the data model to the preparation for a meeting is the pre-meeting class, characterized by a title, a description and a deadline. The group members of a pre-meeting compose the participant class, characterized by user-id, name, affiliation, status and e-mail address. A subclass of the participant class is the coordinator class, representing the participant assigned to the coordination of the pre-meeting.

General topics that are part of a meeting agenda, supposed to be discussed in the pre-meeting, compose the item class, characterized by name, description, creation-date and priority. A goal pursued by the group as part of each item of the meeting agenda defines the objective class, with attributes name, description, status, creation-date and priority.

Assertions made by group members as part of a pre-meeting discussion are represented by the element class, characterized by type, creation-date and content. The element is a generalization of the classes: issue, position, argument, task and remark.

Assertions expressing facts are represented by the pre-decision class, characterized by a name, a description and a creation-date. It results from decisions or common agreements on a subject occurred outside the pre-meeting discussions. A pre-decision is not to be questioned and should work as an assumption during the pre-meeting. It can specialize to a constraint class, representing a restriction to be considered during a discussion.

A reference material for consultation of group members defines the infobase class, with attributes: file-name, directory and supporting application. It points to a repository of information generated by some application, including a previous pre-meeting discussion.

\subsection{Description of relationships between objects}

A pre-meeting is composed by agenda items and the group of participants, where one of them coordinates the pre-meeting, and it assumes pre-decisions or constraints to be considered during the pre-meeting. Items have objectives to be pursued. During the pre-meeting, a participant presents an element that represents an assertion to the discussion.

An element relates-to an objective, and may be an issue, a position, an argument, a task or a remark. An issue may substitute or generate other issues. A position responds-to an issue. An argument is presented either in-favor-of or against a position. A participant may support a position presented by another participant. A participant can make a remark that refers-to an element.

A proposal may originate a task. A participant may candidate-to a task. When candidating he has to tell the system why he is doing it and the deadline he proposes to accomplish the task. If accepted the participant will be responsible-for the task.

To motivate the pre-meeting discussion a participant may reward another participant, informing the reasons to do so.

\subsection{Definition of constraints}

Some constraints are the following. A task can only be presented by the coordinator, a participant cannot reward himself, an argument has to be either in-favor-of or against the same position, a 
participant cannot support a position he presented and only one coordinator can exist in a premeeting.

\subsection{Description of operations}

The objective here is to describe services which are provided to users of the system in order to run a pre-meeting session. Some services may not be required if the pre-meeting does not involve certain functions of the system. An interface should be designed for each type of service. There are also internal services. Services of the latter type are performed by the system but they are not visible to the user; they are not listed in this paper.

An insert function is associated to each object of the system. This can be considered a basic function of the system. The semantics associated to each function, however, is different for each service.

The first service of the system is the support to create a pre-meeting. A user requests the creation of a new pre-meeting to the system that responds to a creation of the database structure that will be the repository of all memory of the pre-meeting. The system also asks the user to define a coordinator of the meeting.

Some of the services available to a coordinator are inserting participants, creating agenda items, creating objectives, delegate coordination (to another participant), changing objective status (open for discussion or closed) and changing deadline of a pre-meeting.

A participant can start a discussion on how to reach an objective and what are the questions to be clarified regarding this objective by inserting issues. Issues are questions or proposals. The operations involved in the discussion process are associating issues (substituting or generating other issues), responding an issue (inserting a position), defining arguments (supporting or opposing positions), supporting positions, inserting remarks (associated to any element) and rewarding participants. A participant has a status, meaning the type of interaction he is prepared to carry out during a period of time and he may change it by doing a "change participant status" operation. Besides informing other participants, the system will use the status information to select events to be notified to each participant.

During the discussion a demand for additional information may occur. This generates a task that will later be assigned to a participant (assigning tasks). The creation of tasks is the responsibility of the coordinator by the creating tasks operation. The participant may only suggest a task, by means of a proposal. If the task is originated by a proposal, the coordinator sets up a corresponding link when inserting the task. A participant may offer himself to carry out a task by the operation candidating to a task. He also informs the deadline he is prepared to accomplish the task and the status of his work (informing the status of tasks).

Originated or not from a task, a participant can create an infobase by the "create an infobase" operation. Infobases store relevant information from other sources, including the memory of a previous pre-meeting. Each infobase has its own supporting application.

A number of views are provided by the system allowing users to access data without having to write a query. Views are different ways to see the objects of the system and can be obtained by doing "accessing views" operations. In the first version of the system, views are fixed, that is, the participant cannot define new views. New versions of the system will incorporate this service. Examples of views provided are listed below.

- List an element with its attributes;

- List an element with its attributes and connections; 
- List of all issues with their positions and arguments, indicating the proponent;

- List of tasks opened with their responsible;

- List of objectives with their issues;

- List a participant with all elements presented by him;

- List of items with their objectives and pre-decisions;

- List of proposals with tasks originated from them.

\section{AN EXAMPLE}

In this section we show the most important concepts of our conversational model in terms of adapting a real example borrowed from Senn (Senn et al., 1991).

Sevco Industries is a company specialized in the design and production of parts and small electronics components. The company was born seven years ago to supply electronic parts to the automotive industry, aircraft, sound equipment, and others. Sevco Industries was founded by John Seversky, who serves now as its president. Harry Jacobson is the operations director, being responsible for the establishment of contacts with possible clients and the follow-up of negotiations between clients and Sevco representatives. He is also responsible for the company's "Costs and Benefits" centers. The leader engineer Jim Olson, in charge of the production operations , together with his two other employees, is responsible of all decisions related to scheduling and purchasing materials and products dispatching. Olson is also responsible for the design of products made by the company. Finally, Marjorie Carbo and her three assistants manage the accounts payables and accounts receivables.

The company grows at an average annual rate of $40 \%$, but it is not always possible to hire appropriate people to match new production requests. The fast growth of Sevco Industries requires the revision of local processing of requests reception and invoicing, currently being done manually. There is a concern of loosing control of requests and accounts receivables as the result of the company growth. Jim Olson asked a study about the reception processes of requests and accounts receivables, used by the company to determine the convenience to automate this functions. Currently, only two employees take care of the requests reception and the control of accounts receivables. The solicitation was done to Joyce Handal, responsible for the Information Systems Department. Nowadays, only the functions of accounts payables and the accountancy of the company are automated.

\subsection{The pre-meeting}

The directors of Sevco Industries resolve to realize a pre-meeting represented as follows, based on the model proposed in this paper. Our purpose here is to identify the entities and relationships of the model, as well as their meanings related to the example. We do not worry, therefore, in showing all the interactions, but just those needed for the understanding of the model. Basic terms are shown in italics.

Jim Olson, assumes the role of coordinator of the pre-meeting, defining the following participants and items :

\section{Participants}

Jim Olson, John Severski, Harry Jacobson, Marjorie Carbo and Joyce Handal. 
Items

- revision of the Requests Reception and Invoicing processes;

- hiring of new employees;

- new possible areas of business for Sevco Industries.

Some pre-decisions have already been taken:

- new employees can only be hired starting next year;

- the company does not intend to work in the microelectronics area;

- no new employees will be hired for the production area;

- the processing of Requests Reception and Invoicing must be done by the same employees.

Next, the group concentrates the attention on the first pre-meeting item: Revision of the processing of Requests Reception and Invoicing.

Jim Olson, as the coordinator, establishes the Objectives of the item in discussion:

- to study the performance of the actual system, based on the operational and expansion plans;

- to verify other experiences of automation;

- to define strategies to improve the Requests Reception and Invoicing processes.

For the first objective, the following issues have been pointed out:

- problems with the actual system, by Joyce Handal;

- effects generated by the problems with the actual system, by Harry Jacobson;

- importance of these effects for the final objective, by Harry Jacobson.

For the first issue, the following positions have been stated:

- We have problems to process all requests, by Jim Olson.

- The customer's information and the requests are not obtained as soon as necessary, by Harry Jacobson.

For the first position, the following arguments in-favor-of are placed (including the one placed by Jim Olson):

- the demand increased too much, by Jim Olson;

- by company policy, workers are not allowed to work overtime, by Marjorie Carbo;

- there are not sufficient employees to do the job, also by Marjorie Carbo.

and the following arguments against:

- we do not have problems, since the job is not being done the right way, by Harry Jacobson;

- at the moment, the requests manipulation occurs in acceptable way, by John Severski.

Analyzing the position and the argument placed early by Harry Jacobson, Jim Olson contributes with a remark :

- I cannot understand your argument considering the position placed by yourself earlier. 


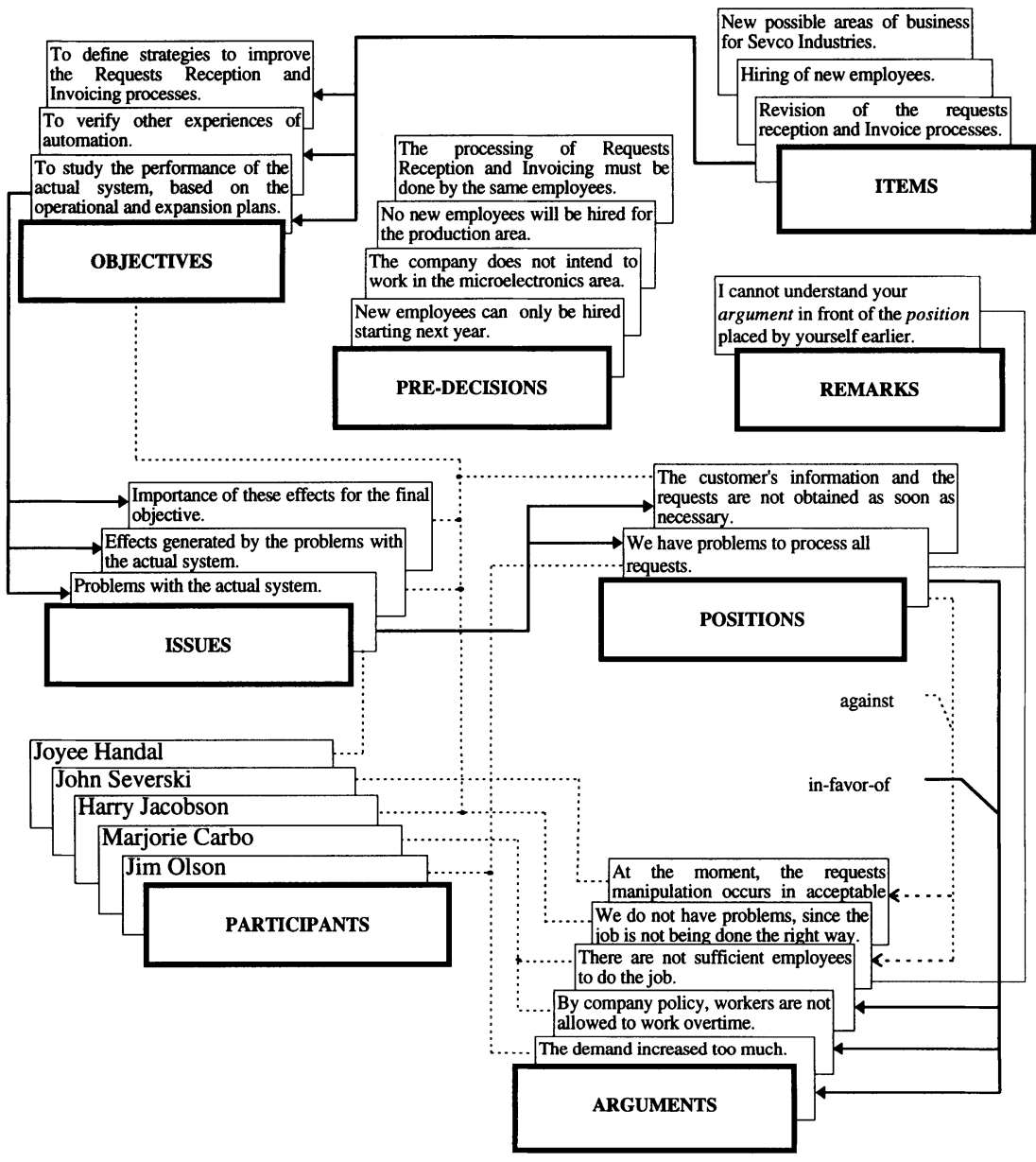

Figure 3 A conceptual view of the interaction using SISCO model.

In Figure 3 we present a conceptual view of the objects and relationships described so far.

Going back to the first Objective, the second issue would be the determination of the effects generated by the problems with the actual system. The positions below are presented:

- there is a customer's claim, by Jim Olson with the argument-in-favor that some requests are lost;

- causes the delay of other tasks, by Marjorie Carbo with the argument-in-favor that when the requests and invoicing are delayed, everybody needs to help to conclude that task. 
Jim Olson's position is supported by Harry Jacobson, who agrees.

For the second Objective, to verify other experiences of automation, one issue is presented:

- are there other systems in the company that were automated, by John Severski.

who substituted it by the next one:

- what are the benefits to the company, obtained by the automated solutions, by John Severski;

- there are some solutions in other companies that could be analyzed, as a proposal that will originate a task, by Joyce Handal.

As responses, the following positions respond-to the proposal made by Joyce Handal:

- I think it is not necessary to look for external solutions, by Harry Jacobson, that concludes that Sevco Industries has a peculiar way to work;

- another company has used automated Requests Reception and Invoicing processing, by Joyce Handal with the argument-in-favor that the obtained results are positive.

Due to this proposal, Jim Olson as coordinator determines the realization of the following task:

- to obtain more information about the automated systems and their results.

Joyce Handal and Marjorie Carbo are candidates to execute the task. Jim Olson, as coordinator chooses Joyce Handal as the person responsible for the task. Joyce Handal will have to collect the information and save it in the INFOBASE, which will contribute to the execution of the task.

John Severski rewards Joyce Handal by her active participation and the quality of the presented issues.

In this way, the pre-meeting continues until the coordinator Jim Olson, determines its end. It is clear that this pre-meeting need not to be performed at the same room and at the same time if a proper software tool such as SISCO is available. Note that even in the same room, participants will have problems following the structure of the pre-meeting. Lack of concentration will very fast slow down the group work. Therefore, SISCO has great advantages in a pre-meeting: it can easily structure the conversation, allowing it to be followed at any time; the system is asynchronous and thus, participants can work on it as they feel or can, augmenting the overall productivity.

Once the pre-meeting is finished, the coordinator can call to a face-to-face meeting to take the decisions. In this way, we believe the meeting will be much shorter and productive than if a classical meeting was called at the beginning.

\section{CONCLUSIONS}

In this article we presented the data model of an information repository to support a pre-meeting interaction. The data model is an extension of the original IBIS model. The model is being used in a project named SISCO that aims to support members of a group preparing for a meeting.

As in IBIS, the evolution of an interaction is represented by objects ordered and shown in a chronological order. The semantics behind this decision is the assumption that a member is aware of the previous objects under the same hierarchy.

Our system's goal is to help the participants to reduce both the equivocality and uncertainty involved in the initial stage of a meeting. A subsequent face-to-face meeting is considered essential and it is not to be replaced by an electronic distributed meeting. The case example shows the complexity of a pre-meeting, which is extremely difficult to follow if proper tools are not available. 
This may explain the lack of meetings performance, where participants loose concentration easily. With a system such as SISCO, we believe that participants will be able to easily interact among themselves and follow a discussion thread, provided an adequate user-interface is built. Since SISCO is a remote and asynchronous system, participants can work at their own pace. Also, they have access to common information and to the records of the pre-meeting.

The actual gain on performance could only be verified if the system is put to work in real-world situations. In order to enable such experiments some prototypes are being developed (Parra and Pino, 1995).

A number of open issues are still under investigation. A simple and intuitive interface, such as the one developed for GTbis (Conklin and Begeman, 1988) is called for. The problem of awareness in asynchronous interactions also requires further research (Borges and Jomier, 1995).

There is already a planned extension to the model. We intend to support hypermedia links and multimedia objects. With hyper links we will be able to point to other objects outside the data model. With multimedia objects we intend to allow group members to illustrate their argumentation with picture, sound and video objects.

\section{Acknowledgments}

This work is partially supported by the Chilean Science and Technology Fund (FONDECYT) grants No. 1950880 and 1940269, by the Brazilian Research Council (CNPq) grant No. 200919/94-6, and grants from RITOS (CYTED), UNESCO-Orcyt, Lotus-Paraguay, Synapsys S.A. and ORACLE Chile. We thank Maria Luiza Campos for her help in the data model.

\section{REFERENCES}

Bellassai, G., Borges, M., Fuller, D., Pino, J.A. and Salgado, A.C. (1995) SISCO: A tool to improve meetings productivity, in Proceedings of the First Cyted-Ritos International Workshop on Groupware, Lisbon, Portugal, 149-161.

Bergmann, N. and Mudge, J.C. (1994) Automated assistance for the telemeeting lifecycle, in Proceedings of the ACM 1994 Conference. on Computer Supported Cooperative Work (CSCW 94), Chapel Hill, N.C., U.S.A., 373-384.

Borges, M. and Jomier, G. (1995) Using database versions to support awareness in group interactions, Proceedings of the Workshop in Version Control at ECSCW'95, Stockholm, Sweden.

Conklin, J. and Begeman, M. (1988) gIBIS: A hypertext tool for exploratory policy discussion. ACM Transactions on Office Information Systems, 6 (3), 303-331.

Daft, R. and Lengel, R. (1986) Organizational information requirements, media richness and structural design. Management Science, 32 (5), 554-571.

Ellis, C.A., Gibbs, S.J. and Rein, G.L. (1991) Groupware - Some issues and experiences. Communications. of the ACM, 34 (1), 39-58.

Hackman, J. and Kaplan, R. (1974) Interventions into group process: An approach to improving the effectiveness of groups. Decision Sciences, 5,. 459-480.

Jay, A. (1976) How to run a meeting. Harvard Business Review, 54 (2), 43-57.

Khoshafian, S. and Buckiewicz, M. (1995) Introduction to groupware, workflow, and workgroup computing. John Wiley and Sons, San Francisco. 
Kunz, W. and Rittel, H. (1970) Issues as elements of information systems, Working Paper \# 131, Institute of Urban and Regional Development, University of California at Berkeley.

Nunamaker, J.F. et al. (1991) Electronic meeting systems to support group work, Communications of the ACM 34 (7), 40-61.

Parra, R. and Pino, J.A. (1995) N-Sisco: A Notes implementation of SISCO, in Proceedings of the First Cyted-Ritos International Workshop on Groupware, Lisbon, 125-137.

Rumbaugh, J, et al. (1991) Object-oriented modeling and design. Prentice Hall Inc., Englewood Cliffs, N.J.

Senn, J. et al. (1991) Analysis and design of information systems. McGraw-Hill, New York

Streitz, N., Geibler, J., Haake, J. and Hol, J. (1994) DOLPHIN: Integrated meeting support across local and remote desktop environments and liveboards, in Proceedings of the ACM 1994 Conference on Computer Supported Cooperative Work (CSCW 94), Chapel Hill, N.C., U.S.A., 345-358.

Wagner, G., Wynne, B. and Mennecke, B. (1993) Group support systems facilities and software, in Group Support Systems - New Perspectives (ed. L. Jessup and J.S. Valacich), MacMillan Publishing, New York, 8-55.

\section{BIOGRAPHY}

Geronimo Bellassai got a B.Sc. in Electromechanical Engineering at the Universidad Nacional de Asuncion, Paraguay in 1982. He specialized in Electronics at the Instituto Girolamo Montani de Fermo, Italy. He currently is Vice Dean and professor at the Universidad Católica de Asuncion (Faculty of Science and Technology). He is also chairman of the university's Laboratory of Digital Electronics.

Marcos Borges obtained a doctorate at the University of East Anglia (UK) in 1986. Since 1975, he is a consulting analyst for the Núcleo de Computação Electrônica of the Universidade Federal de Rio de Janeiro (UFRJ) and an Associate Professor at the Department of Computer Science of the same university. He has been a consultant for several companies in Brazil and in US. He is currently spending a sabbatical at Santa Clara University, California, USA.

David Fuller is professor in computer science at Pontificia Universidad Católica de Chile. His Computer Science Doctorate was obtained at the Imperial College of Science and Technology, UK in 1989. He currently is the Treasurer of the Chilean Computer Science Society and Executive Secretary of CLEI (Latin American Association of Academicians and Professionals).

Jose Alberto Pino obtained a M.S. and qualification towards a Ph.D. degree at the University of Michigan (USA) in 1977. He is a Professor at the University of Chile (Computer Science Dept.). He is a member of IFIP TC13 (Human Computer Interaction) and has participated in international Editorial Committees and Program Committees (such as the HCI Conference 93 and IFIP World Congress 92).

Ana Carolina Salgado obtained her Doctorate from the University of Nice (France) in 1988. She is Associate Professor at the Universidade Federal de Pernambuco, Brazil (Department of Informatics). She is the Coordinator of Undergraduate Studies in Computer Science at her university. 